\title{
Looking to the future
}

Citation for published version (APA):

Atkinson, M. J., Stock, N. M., Alleva, J. M., Jankowski, G. S., Piran, N., Riley, S., Calogero, R., Clarke, A., Rumsey, N., Slater, A., Diedrichs, P. C., \& Williamson, H. (2020). Looking to the future: Priorities for translating research to impact in the field of appearance and body image. Body Image, 32, 53-61. https://doi.org/10.1016/j.bodyim.2019.10.006

\section{Document status and date:}

Published: 01/03/2020

DOI:

10.1016/j.bodyim.2019.10.006

Document Version:

Publisher's PDF, also known as Version of record

\section{Document license:}

Taverne

\section{Please check the document version of this publication:}

- A submitted manuscript is the version of the article upon submission and before peer-review. There can be important differences between the submitted version and the official published version of record.

People interested in the research are advised to contact the author for the final version of the publication, or visit the DOI to the publisher's website.

- The final author version and the galley proof are versions of the publication after peer review.

- The final published version features the final layout of the paper including the volume, issue and page numbers.

Link to publication

\footnotetext{
General rights rights.

- You may freely distribute the URL identifying the publication in the public portal. please follow below link for the End User Agreement:

www.umlib.nl/taverne-license

Take down policy

If you believe that this document breaches copyright please contact us at:

repository@maastrichtuniversity.nl

providing details and we will investigate your claim.
}

Copyright and moral rights for the publications made accessible in the public portal are retained by the authors and/or other copyright owners and it is a condition of accessing publications that users recognise and abide by the legal requirements associated with these

- Users may download and print one copy of any publication from the public portal for the purpose of private study or research.

- You may not further distribute the material or use it for any profit-making activity or commercial gain

If the publication is distributed under the terms of Article $25 \mathrm{fa}$ of the Dutch Copyright Act, indicated by the "Taverne" license above, 


\title{
Looking to the future: Priorities for translating research to impact in the field of appearance and body image
}

\author{
Melissa J. Atkinson ${ }^{\mathrm{a}, \mathrm{b}, *}$, Nicola M. Stock ${ }^{\mathrm{b}}$, Jessica M. Alleva ${ }^{\mathrm{c}}$, Glen S. Jankowski ${ }^{\mathrm{d}}$, \\ Niva Piran ${ }^{\mathrm{e}}$, Sarah Riley ${ }^{\mathrm{f}}$, Rachel Calogerog ${ }^{\mathrm{g}}$, Alex Clarke ${ }^{\mathrm{b}}$, Nichola Rumsey ${ }^{\mathrm{b}}$, Amy Slater $^{\mathrm{b}}$, \\ Phillippa C. Diedrichs ${ }^{\mathrm{b}}$, Heidi Williamson ${ }^{\mathrm{b}}$ \\ a Department of Psychology, University of Bath, UK \\ ${ }^{\mathrm{b}}$ Centre for Appearance Research, University of the West of England, UK \\ ${ }^{c}$ Department of Clinical Psychological Science, Maastricht University, The Netherlands \\ d School of Social Sciences, Leeds Beckett University, UK \\ e Department of Applied Psychology and Human Development, University of Toronto, Canada \\ ${ }^{\mathrm{f}}$ School of Psychology, Massey University, New Zealand \\ ${ }^{g}$ Department of Psychology, Western University, Canada
}

\section{A R T I C L E I N F O}

Article history:

Received 14 August 2018

Received in revised form 18 October 2019

Accepted 18 October 2019

Available online 29 November 2019

Keywords:

Body image

Appearance

Research priorities

Collaboration

Impact

Activism

\begin{abstract}
A B S T R A C T
The field of body image and appearance research and practice is progressing; however, there is still work to be done to ensure broad societal impact. This article consolidates reflections from a range of established and early career experts in the field of appearance and body image, with a focus on stimulating and guiding future agenda setting and translation from research to impact. We conducted a thematic analysis of transcripts from nine recorded 5-minute presentations, delivered by researchers and clinicians as part of a special invited presentation session at a biennial international conference, 'Appearance Matters,' in the UK. Four themes were identified: Moving Beyond the Individual; Consolidation and Collaboration; Commitment to Implementation; and Positive and Protective Frameworks. These themes are discussed alongside recommendations for researchers and practitioners working in these fields to advance research, advocacy, and impact outside of academia.
\end{abstract}

(c) 2019 Elsevier Ltd. All rights reserved.

\section{Introduction}

Significant progress has been made in the field of body image and appearance research in recent decades. Broadly, research has advanced knowledge and understanding of the multidimensional nature of body image and appearance, key factors and influences in their development and maintenance, and the creation and evaluation of a range of assessments and interventions in various settings and populations (Cash \& Smolak, 2011; Rumsey \& Harcourt, 2012; Tylka \& Piran, 2019). This body of work has formed an important foundation from which to guide ongoing research, clinical practice, and advocacy efforts, as well as to stimulate ongoing research. Given the increasing recognition being paid to the importance of body image and appearance at a societal level (e.g., the UK All Party Parliamentary Group report on Body Image, 2012;

\footnotetext{
* Corresponding author at: Department of Psychology, University of Bath, Bath, BA2 7AY, UK.

E-mail address: m.j.atkinson@bath.ac.uk (M.J. Atkinson).
}

Nuffield Council on Bioethics, 2017), as well as the proliferation of associated research ${ }^{1}$, it is important that we continue to move forward in a strategic manner in order to engender meaningful progress.

In service of this aim, a panel of established and early career experts in the field were invited to give succinct 5-minute presentations to answer the following question at an international biennial conference in the field of appearance and body image, Appearance Matters 7, held in 2016: 'What is the most important priority for the field of appearance and body image research and practice in the next five years and why?' This session was a new addition to the scientific programme, aimed at providing a boost of energy and stimulation to close the proceedings. Ultimately, it also provided a unique opportunity to crystallise expert perspectives regarding important directions for our field, offering a

\footnotetext{
${ }^{1}$ For example, a search using the term "body image" conducted in PsycINFO on $7 / 12 / 17$ returned the following number of hits in preceding decades: 1985-1995 = $2479 ; 1996-2006=4776 ; 2007-2017=8909$
} 
valuable springboard for researchers and clinicians considering next steps. Across speakers, a number of common threads were noted, with a particular focus on prioritising efforts relating to maximising societal impact. Although only one approach to facilitating progress in the field, it nevertheless represents an important one. The aim of this article was to consolidate reflections from these established and early career experts, and to offer directions for meaningful progress with particular respect to the translation of research into impact in the field of body image and appearance.

\section{Method}

Five established experts and four early career researchers based in institutions from the UK, Canada, and the Netherlands (first nine authors) participated in an invited presentation session at the Appearance Matters 7 conference in London, June 2016 (www.uwe. ac.uk/appearancematters). They were selected by the conference co-chairs (last three authors) for having an established or emerging presence in the field of appearance and body image, across different areas of expertise, and who had submitted an abstract to attend and present at the conference. Presenters were predominantly women ( 8 women, 1 man). Established experts reflected an average 26.5 years (range 18-38) of experience in the field, while early career researchers reflected an average 5.5 years of experience (range 5-6). The presenters ranged in backgrounds and perspectives, encompassing research and clinical expertise in body image and appearance as related to: media and sociocultural influences, eating disorder prevention, visible difference (such as may be present from birth e.g., a cleft lip and palate, or acquired later in life either through illness or injury e.g., a burn, a skin condition, or as a consequence of cancer treatment), women's health, masculinity, feminism and postfeminism, critical psychology, objectification, sexualisation, weight stigma, positive embodiment, and gender socialisation. While not specifically selected for, we note that many of the speakers reflected an applied research focus, and a social justice viewpoint (defined here as "the goal to decrease human suffering and to promote human values of equality and justice"; Louis, Mavor, La Macchia, \& Amiot, 2014), which provides context for the ensuing analysis and discussion.

Each 5-minute presentation was accompanied by predominantly visual slides, and all were audio recorded. Full-length audio recordings of the presentations are available in Episode 12 of 'Appearance Matters: The Podcast' (http://soundcloud.com/ appearance-matters). All speakers retrospectively consented to audio recording and release on the podcast, transcription and subsequent thematic analysis, and use of quotes in the present paper.

Audio recordings were transcribed verbatim and subjected to inductive thematic analysis, following the steps outlined in Braun and Clarke (2006): (1) familiarise yourself with the data; (2) generate initial codes; (3) search for themes; (4) review themes; (5) define and name themes; and (6) produce the report. Analysis was seen as a recursive process, and detailed notes were written throughout. Themes were subsequently chosen for their prevalence and/or their importance (or "keyness") in relation to the research question. All transcripts were analysed by the first and second authors/speakers: both female early career researchers who participated as presenters in the session themselves, adopt an applied research focus and social justice viewpoint as noted above, represent different areas of the field (body image/eating disorder prevention and cleft lip and palate, respectively), and have experience in conducting qualitative analysis. Transcripts were reviewed by both authors independently. Initial coding reached an average agreement of $92 \%$ (range $82 \%-100 \%$ ). Minor discrepancies were discussed until full agreement was reached and themes, including wording, could be finalised. The resultant themes were shared with all speakers and conference co-chairs (all authors) to ensure unbiased reporting and establish the accuracy of the analysis, given the positionality of the first two authors as both presenters and those conducting analysis, and to provide any additional feedback for incorporation into the discussion.

\section{Results}

Four themes were identified: (a) Moving Beyond the Individual; (b) Consolidation and Collaboration; (c) Commitment to Implementation; and (d) Positive and Protective Frameworks. Each theme is outlined below using exemplar quotes.

\subsection{Moving beyond the individual}

The first theme referred to the continuing trend for appearance related interventions to be targeted at the individual level, and calls for future work to focus more on overarching systems. Several speakers described the ways in which society still typically places the onus on the individual.

[We live in] an economy that requires its citizens to...transform themselves to meet the changing needs of a market... we have the idea that both health and weight are outcomes of individual lifestyle choices and risk management...women are expected to work on their bodies. . constructed as choiceful and empowering - (expert).

One speaker also highlighted the ways in which language, embedded within culture and society, reinforces these ideas.

Social ideologies and structures of power directly shape embodied lives, such that cultural texts are inscribed into and onto bodies....skirt-length alone [ranges from]. .. 'matronly' and 'prudish' to 'flirty' and 'asking for it'. . . These inscribed social labels reflect adverse cultural ideologies - (expert).

Many speakers therefore outlined how research has traditionally focused on individual change as the focal point.

We try to correct [individuals'] faulty attitudes and beliefs and behaviours...We have looked at their immediate context; peers, family members, friends, parents, and tried to change other people.... These haven't met with the success that we have hoped...partly because both are placing blame on individuals (early career).

Given the moderate success of individualistic approaches to date, almost all speakers spoke in support of a greater move towards targeting broader systems and industries. Yet, the many challenges of such a move were acknowledged.

There are so many strong forces. . . Big businesses are in it for lots of money, and governments are afraid to act even though countless inquiries have said that they need to... It's led a lot of us...to occasional points of despair - (expert).

To advance the field, a minority of speakers encouraged researchers and clinicians to push for a broader critical 'reading' of the body.

Reading social texts on bodies...[will ensure that] our etiological theories are more comprehensive. ..will deepen the understanding of embodied distress. . . will align our interventions more. . .with goals of social justice and equity - (expert).

There was also a common focus on engagement in activism to raise awareness and action at a community level.

A key priority for the field is to tie our research more closely to activism. . . Activism builds partnerships. . .is about connection and 
it's about community. It is empowering for individuals and groups, and it's fundamental to social change - (expert).

Finally, several speakers noted the need to engage more effectively with influential parties.

[We need to] understand how communication works, how influence works, how persuasion works, and we need to learn how to work using the agendas of these big, powerful partners that we've now acquired - (expert).

\subsection{Consolidation and collaboration}

This theme calls for the consolidation of constructs and methods currently utilised within the field, and closer collaboration at all stages of research and practice. Two speakers identified one of the key challenges of current research to be an ongoing focus on the intricacies of modern theories, models, and measures.

As [Toffler] said in 1981, very aptly, 'obsessive emphasis on quantified detail without context, on progressively finer and finer measurement of smaller and smaller problems, leaves us knowing more and more about less and less' - (early career).

Further, several speakers commented on the number of different constructs and terms being utilised within current research and practice.

We drown newcomers to the field in a ridiculous plethora of constructs. Not only that, but. . .we talk about very similar things, but we call them by different names - (expert).

One speaker described how this has led to the application of different methodological approaches, resulting in findings that cannot be easily compared.

In our attempts to capture a comprehensive understanding of adjustment, we've applied a multitude of different measures, at different time points, and with different populations - (early career).

Consequently, a majority of speakers called for researchers to work more closely with each other and a range of stakeholders, to achieve consensus in relation to constructs and methods.

We need broad, overarching frameworks, which encompass both generic and condition-specific aspects of adjustment. . .We need to agree upon an effective set of appropriate measures, and then utilise those measures consistently over time and across teams. .. We need qualitative studies and patient and public involvement exercises to corroborate the relevance and acceptability of these frameworks and measures - (early career).

Additionally, collaboration was described by almost all speakers as essential for ensuring impactful interventions.

We need teamwork at all stages of our intervention development, evaluation, dissemination. . from the end users in co-creating the materials, to collaborating with community and industry partners, and involving advocacy groups and public figures... to move towards an integrated and coordinated approach... across all development periods, all access points and around the globe (early career).

Finally, two speakers strongly promoted the integration of research participation into clinical practice.

Ultimately, I believe the answer. . .lies in our ability to integrate research much more fully into practice, so that taking part in research becomes the norm for patients, rather than the exception - (early career).

\subsection{Commitment to implementation}

This theme encompassed the need for an increased focus on implementation - the translation of research findings and interventions into practice - throughout the entire research process.

Many speakers highlighted the gap that still exists between research and practice, and the need for researchers to go beyond simply highlighting implications and recommendations in end-ofproject publications and presentations.

That wonderful phrase 'these findings have implications for clinical practice' - but I'm not actually going to tell you what they are... I'd like us to think about redefining implementation rather than dissemination as the end point of research - (expert).

Therefore, several speakers called for integrated research planning, ensuring that interventions are developed and designed with implementation in mind from the beginning, alongside a plan for delivering the intervention to end users.

We need to be prioritising implementation and dissemination from the outset, preferably on a large scale and with minimal cost...we need to make sure that our interventions are packaged in a way that's attractive...to adopt formats that are current and userfriendly, [to] maximise. . buy-in and engagement - (early career).

Nevertheless, the practical necessities of carrying out successful implementation were highlighted, with one speaker calling for funders to provide resourcing for implementation plans.

I'd like to see funders set targets for supporting positive research findings into the implementation phase. The difficult bit. . is not getting the initial money to show and demonstrate that an intervention works, it's that next phase when you want to get it into clinical practice - (expert).

Several speakers felt that advocacy and activism were also important for facilitating research implementation and impact.

Impact validity, which refers to the extent to which research has the potential to play a role in social and political change...is just as important as the traditional criteria for evaluating the rigour of research... we should...work on creative ways of bringing our research to the stakeholders' table with whatever resources and networks we have at our disposal - (expert).

However, there was also a recognition by a minority of speakers that specific training in implementation and activism should be provided alongside the development of traditional research skills.

We've really got to think about the training we provide for early career and mid-career researchers, and also for [senior researchers], because it is hard for us to get out of our boxes, it is hard for us to learn new skills - (expert).

\subsection{Positive and protective frameworks}

The final theme reflects the importance of a broader conceptualisation of body image and resulting interventions, incorporating greater emphasis on positive and protective factors.

In the first instance, many speakers highlighted the need to broaden perspectives of the body to include more than just appearance, in order to deepen our theoretical understanding and create a more holistic view of the body.

An emerging trend in the field has involved examining a broader range of ways of inhabiting the body, such as functionality, agency, and attunement, and with that, positive ways of inhabiting the body. These shifts will inform our etiological developmental models, as well as our interventions - (expert). 
[We] forget that the body and therefore body image is not only [about] appearance but also function... This bias. . could be limiting our scope of vision and inspiration in our research. - (early career).

The broader benefits of incorporating a positive and protective approach within intervention development and evaluation were also highlighted by several speakers, to capture benefits that are potentially being missed when only targeting and assessing negative risk factors.

Part of the problem [with establishing efficacy] is that we are applying a risk factor framework... [We need to also be] evaluating protective factors. . We need a better understanding of how [protective factors] do promote resilience, how they work together, and how they interact with risk factors - (early career).

Social activism was also presented by one speaker as an avenue that helps to promote benefits at both an individual and societal level, through a positive and empowering lens.

Activism can influence appearance factors and be a powerful tool for healing... [e.g., engaging] youth as activists. . .and body ambassadors who have gone on to transform cultural attitudes and...lobby for anti-discrimination policies in local schools and beyond. Activism in this context shifts the emphasis away from raising their self-esteem to increasing their power and to finding their individual and collective voice, both of which are lost through objectification and oppression - (expert).

\section{Discussion}

The aim of this article was to consolidate reflections from established and early career experts on important priority areas for shaping future application and translation of research to impact in the field of body image and appearance. Across nine presentations from speakers with experience in body image and appearance research and from a range of backgrounds, four common themes were identified by the first two authors/speakers: (a) Moving Beyond the Individual; (b) Consolidation and Collaboration; (c) Commitment to Implementation; and (d) Positive and Protective Frameworks. Taken together, these themes coalesce into a message for researchers and clinicians to 'think bigger' - to expand and broaden their focus and field of vision for progress and impact. The following discussion elaborates on how to 'think big' within the context of each of the themes identified and offers a number of recommendations for guiding research application and translation to encourage broad impact (Table 1 ).

\subsection{Think big: moving beyond the individual}

This first theme emphasised the need to expand the focus from the individual to the wider social and cultural systems that shape the individual. While recognising sociocultural influences on appearance and body image is not new to the field (Piran, Levine, \& Steiner-Adair, 1999; Thompson, Heinberg, Altabe, \& TantleffDunn, 1999), the importance of making this shift is perhaps more pertinent than ever. The subjugating social and cultural ideologies that still exist around bodies and appearance (Calogero \& Jost, 2011; Calogero \& Tylka, 2014; Piran \& Teall, 2012), coupled with the rise and ubiquity of social media and associated consequences (e.g., Fardouly, Diedrichs, Vartanian, \& Halliwell, 2015; Holland \& Tiggemann, 2016; Lonergan et al., 2019; Mills, Musto, Williams, \& Tiggemann, 2018; Tiggemann, Hayden, Brown, \& Veldhuis, 2018), contribute to a seemingly insurmountable challenge for researchers and clinicians. What can be done? The findings of the current study highlight the need for greater and consis- tent efforts at targeting overarching social systems across the field, along two related pathways.

First, there is a need to better incorporate a broader social critical analysis into etiological frameworks. Sociocultural models of eating disorder development (e.g., Thompson et al., 1999) provide a good foundation, with recent theoretical work also providing comprehensive consideration of the multiple social forces and ideologies inscribed into and onto bodies, validate individuals' experiences within the context of their lives, and foster greater embodiment (Piran, 2017; Riley, Evans, \& Robson, 2018). Encouraging etiological frameworks (and resulting language) that moderates individual responsibility and blame for appearance is likely to reduce anxiety, comparison with others, and self- and social judgement (Riley, Evans, \& Mackiewicz, 2016). Furthermore, recent work has shown that consumers themselves feel that a broadening of the discourse related to appearance in society and the media (e.g., greater diversity in representation, shifting focus from appearance to function and health) is likely to be more effective at reducing appearance-related concerns than strategies aiming to change individual processes, beliefs, and attitudes (Paraskeva, Lewis-Smith, \& Diedrichs, 2017). There remains a need to bring greater recognition to seeing body image concerns as socially produced and not individual pathologies.

Second, there is a need to engage key players and stakeholders with existing power and influence in shaping society - consistently and en masse. This engagement includes challenging profit imperatives and encouraging greater corporate responsibility in businesses and companies that play an instrumental role in shaping unrealistic appearance ideals. This may concern businesses that have not yet committed to corporate responsibility, as well as to supporting and guiding those who have. It also includes regularly lobbying and engaging government ministers, lawyers, and social policy makers within the political system with concrete evidence regarding the consequences of their actions and which contribute to their own objectives, as well as viable strategies for translating research findings into law and policy, such as has been highlighted by Austin (2015). Going beyond the individual also means regularly working with other large social institutions such as the educational, social welfare, and health systems. Finally, engaging influencers also includes embracing and encouraging activism such as volunteerism and public engagement, to drive social change that will benefit communities and societies at large, as well as empowering and healing individuals (Calogero, 2013). Advocacy and activism should be adopted universally as a worthwhile companion to science, ensuring that research is used to drive real social change and demonstrate impact validity - defined as "the extent to which research has the potential to play a role in social and political change, or is useful as a tool for advocacy or activism" (Massey \& Barreras, 2013, p. 616). While recognising that all these types of activities do not traditionally fall within the researcher or practitioner skill set, we, at least, encourage strategic research to provide relevant evidence for such initiatives, and at most, encourage actively seeking additional training, partnerships, and use of existing frameworks (e.g., Austin, 2015) to increase influence.

As the speakers identified, research, activism, and partnerships with stakeholders may not always be harmonious, and researchers may take different stances. For instance, researchers' goals for reducing appearance dissatisfaction, juxtaposed with the profit imperatives (and the premise this is built on) of big business, provides a seeming source of tension requiring careful consideration (Craddock, Ramsey, Spotswood, Halliwell, \& Diedrichs, 2019; Jankowski, 2016). However, recognising that there is a financial, moral, and social imperative for businesses to operate in ways that add value to society beyond economic gains for shareholders (Pfitzer, Bockstette, \& Stamp, 2013; Porter \& Kramer, 2006) opens up unique opportunities for researchers and advocates to 
Table 1

Recommendations for encouraging progress in the translation of body image and appearance research into impact across the four themes.

Theme 1: Moving Beyond the Individual (for researchers and practitioners)

- Adopt, or refine, theoretical models that incorporate social and systemic influences to underpin and guide research and practice e.g., the Developmental Theory of Embodiment (Piran \& Piran \& Teall, 2012).

- Challenge social discourse that blames individuals when interacting with colleagues, participants, stakeholders, patients/clients (for guiding work, see Gill, 2007; McVey et al., 2013; Riley et al., 2008, 2018).

- Increase development, evaluation, and implementation of interventions aimed at macro (community, societal, environmental, and policy) level change (e.g., reforming workplace dress and appearance codes; regulation of adverts that irresponsibly airbrush or exaggerate effects of appearance-based products/services; providing and engaging in professional training aimed at reducing stigma based on weight, visible differences, or other appearance-based feature; greater public education enhancing understanding of embodied justice).

- For researchers, design and conduct research with direct relevance and applicability for policy decisions and/or business practices, and seek to partner with practitioners, advocacy organisations, politicians, and legal representatives to initiate and shape proposals which maximise success for legislative change (e.g., see Austin, 2015; Record \& Austin, 2016).

- Engage in advocacy and activism to challenge damaging cultural norms and practices, including sharing of research findings more broadly (e.g., reports, infographics, and summaries - clinicians/practitioners are also encouraged to support academics in sharing); engaging in public and policy exchange events as a researcher or practitioner; creating/sharing content on social media and other public outlets; writing articles for a broad audience, including opinion pieces, blogs, Letters to the Editor, articles in popular science magazines, and open letters; participating in media interviews for a broad audience; organise, support, and/or participate in protests, petition campaigns, and other advocacy campaigns (e.g., see the work of Endangered Bodies, https://www.endangeredbodies.org). It is also important to note that activism can be enacted in small, daily acts, such as speaking out when a colleague makes an inappropriate appearance-based comment, discussing the importance of activism in lectures, speaking positively about one's own body rather than engaging in 'fat talk,' tweeting disapproval of stigma/approval of diversity; using non-stigmatising language with participants, patients/clients, etc.

- For researchers, make proposals to academic institutions for encouraging, recognising, and rewarding engagement, advocacy, and other activities aimed at translating research to impact (e.g., providing a set protected percentage of workload for such activities, and offering training and development opportunities to ensure effective, efficient and impactful efforts).

Theme 2: Consolidation and Collaboration (for researchers)

- Conduct thorough literature reviews and routinely make requests via personal and professional/academic networks (e.g., ResearchGate, professional member organisations, listservs) during research design phase to avoid overlap and encourage collaboration.

- Make research protocols, materials, interventions, and data publicly accessible according to open science practices (Open Science Collaboration, 2015), via existing pre-registration and data sharing services (e.g., the Open Science Framework, https://osf.io) or generation of a field-specific portal for sharing and accessing such information.

- Identify working parties for different areas within the field (e.g., assessment, interventions) to enhance a coordinated approach and more collaborative research. For example, the COST Action Network IS1210 ("Appearance Matters: Tackling the Physical and Psychosocial Consequences of Dissatisfaction with Appearance"; COST Action, 2017) brought together 178 scholars from 36 countries via meetings in various countries to provide a knowledge sharing platform and stimulate collaborative and coordinated progress. Although this has now concluded, the biannual Appearance Matters conference could provide an ongoing platform for "working groups" with similar aims and interests, each setting priorities and plans for action between conferences. In order to facilitate and not hinder progress, a clear purpose and set of objectives would be necessary.

- Outline key constructs within specific areas of the field, identifying current best practice for their measurement, and offering recommendations for future research and practice (e.g., Tylka \& Wood-Barcalow, 2015; Webb et al., 2015). Such guidelines could be formulated based on current 'gold standards' for how constructs can be manipulated or targeted within experimental settings and intervention programmes, take a cross-disciplinary perspective (e.g., what can we learn from other areas both within and external to the field), and be updated at appropriate intervals.

- Recognise and raise awareness of institutional pressures for academics, and be more vocal in supporting or initiating calls for 'slow scholarship' at a governance level including more mindful research, an ethics of care for each other and our participants, and more genuinely collaborative partnerships whose end point is not always a high impact publication but meaningful research that tangibly benefits participants and wider society (Hartman \& Darab, 2012).

Theme 3: Commitment to Implementation (for researchers and practitioners)

- Actively seek ongoing partnerships between researchers, practitioners, and stakeholders (e.g., clinical services, schools, community organisations, businesses), with regular meetings to discuss research, exchange priorities and ideas, and generate future directions.

- Encourage principles of behaviour change and motivational frameworks to encourage bi-directional participation in research at all levels of practice (e.g., users, lived experience, practitioners/service providers, governing organisations/services).

- For researchers, clearly inform stakeholders on the process, benefits, and findings, of research in an accessible way (e.g., infographics, short videos, lay reports) and engage them from the outset to co-produce and implement evidence-based research and practice, considering guidance from existing frameworks e.g., Mixed Methods application in Action Research framework (MMAR; Ivankova, 2014), community-partnership research (CSR; e.g., Becker et al., 2009, 2017), triggers-to-action framework (Austin, 2015).

- Include implementation science as part of core teaching for research and vocational training (e.g., clinical and healthy psychology, allied health professions, medicine and nursing) from undergraduate through to postgraduate, and within training opportunities for established academics, practitioners and relevant professionals.

- For researchers, consider implementation as the end-point of the research, and explore potential barriers as a routine part of research design, proposals and grant applications, informed by public involvement from users and stakeholders.

- For academics, lobbying academic institutions and funding bodies on the value of implementation, the need to broaden the reward structure and criteria for success from the focus on publications, citations and impact factors (as this disincentivises implementation efforts due to the time involved that may take away from publication output) e.g., proposals and petitions for implementation-focused funding calls, explicitly making arguments for this within relevant grant applications, leveraging authority as grant reviewers and members of governing bodies and committees).

Theme 4: Positive and Protective Frameworks (for researchers and practitioners)

- Adopt a broad conceptualisation of the body (e.g., body functionality, embodiment, intuitive and adaptive eating and self-care; see Tylka \& Iannantuono, 2016; Tylka \& Piran, 2019) in etiological and epidemiological research, when theorising and modelling constructs, in developing and evaluating interventions, and in clinical practice.

- For researchers, explore longitudinal mediational and interactional models that include both positive and negative factors, to further our theoretical understanding and identify key intervention targets. Initially, we recommend reviewing and refining existing models (e.g., for body image, consider the Developmental Theory of Embodiment [Piran, 2017; Piran \& Teall, 2012], the Acceptance Model of Intuitive Eating [Avalos \& Tylka, 2006], the Attuned Representational Model of Self [Cook-Cottone, 2006], the Gratitude Model of Body Appreciation [Homan \& Tylka, 2018]), and then if necessary, consider proposing new models (see Pennesi \& Wade. 2016 for discussion on this point with relevance to eating disorders).

- More frequent inclusion of positive and protective outcomes in evaluating prevention and treatment within research trials and patient/service evaluation, rather than a singular focus on reducing risk factors/symptoms.

- Assess, harness, develop, and evaluate existing and novel positive influences on social norms and popular culture regarding bodies and appearance, including (but not limited to) social media content (e.g., Cohen, Irwin, Newton-John, \& Slater, 2019; Fardouly \& Rapee, 2019; Slater, Cole, \& Fardouly, 2019, 2017; Tiggemann \& Zinoviev, 2019), public awareness campaigns (e.g., Mulgrew et al., 2019; Rodgers, Kruger, Lowy, Long, \& Richard, 2019), media advertising (Craddock et al., 2019), and education in schools. 
cautiously, transparently, and strategically engage with businesses to affect positive social change at a systems-level (e.g., Becker et al., 2017). In short, researchers are exhorted to think outside the traditional research box and develop skills and strategies to both challenge the discourse around body image and develop and utilise stakeholder partnerships, to facilitate macro level impact and spread that will drive real social change.

\subsection{Think big: consolidation and collaboration}

The second theme highlights a call for consolidation and collaboration across the field, and a move away from research efforts which results in an increasing plethora of constructs, measures, and interventions, with no demonstrable gain. While a "splitting hairs" approach is understandable given the desire to capture comprehensive and multifaceted issues, and a desire or pressure to put one's name to something new, it raises a considerable barrier to drawing meaningful conclusions and therefore to implementing evidencebased practice (even at a local level, let alone global scale). How should this difficult challenge be tackled? The current findings call for efforts to prioritise consensus among research and stakeholder groups on constructs, measures, and intervention pathways, and to challenge the academic culture in which there exists a pressure to constantly innovate.

Consolidating constructs into broad, overarching frameworks that encompass well-defined generic and condition-specific constructs has already been identified as crucial for guiding research and practice in the area of cleft lip and/or palate (Stock et al., 2016). This approach could be adopted for other areas of body image and appearance, with collaborative agreement on the most useful theories and constructs and how they interrelate, and then how they can be measured consistently across applications to enable welljustified conclusions, and inform policy and practice in a timely manner. As an example, Tylka and Wood-Barcalow (2015) have clearly delineated what positive body image is, and what it is not and Webb, Wood-Barcalow, and Tylka (2015) have outlined key measures to assess the various aspects of positive body image. This exemplifies productive efforts to clarify key constructs and how they can be effectively measured, providing clear direction for future research and application. To facilitate and not hinder progress, such an approach requires clear purpose and objectives, and an investment in communication and sharing of ideas and resources between research groups over and above individual achievements.

Regarding intervention pathways, unless there is clear and compelling justification, researchers are cautioned against (even inadvertently) re-inventing the wheel, primarily referring to the creation of independent interventions that do not provide anything substantially new. Instead, we urge extensive reviews prior to intervention development via published work and professional networks, as well as closer collaboration with colleagues working in overlapping areas, to consolidate and refine existing knowledge and programmes in a strategic manner, ultimately aiming for an integrated and coordinated approach to intervention strategies across developmental periods and access points (e.g., Yager, Diedrichs, Ricciardelli, \& Halliwell, 2013). The goal of achieving consistency in theoretical frameworks, measures, and intervention pathways around the world should be a focus, while remaining intersectional and sensitive to differences in language, culture, and resource. Given the predominance of research conducted in homogenous majority samples, it is recognised there is insufficient intersectional research to confidently forge ahead with consolidating and disseminating across the board. The authors support calls to investigate theories, measures, and interventions in different groups (e.g., individuals of different gender orientations, ages, ethnicities, sexual orientations, physical abilities, etc.), consistent with the first theme: highlighting another way to move beyond the individual and recognise the various social and systems-level factors that shape experience and intersect with body image and appearance.

\subsection{Think big: commitment to implementation}

The third theme highlighted the need for increased efforts to ensure research has real-world impact. Many in the field would agree that the ultimate goal for appearance research is to drive positive change. Yet despite two decades of growth in evidence-based intervention research, implementation in social and clinical settings (and the establishment of related impacts such as sustained long-term improvement in health outcomes, cost-effectiveness, and policy change) is largely still playing catch-up (Diedrichs, 2016). The first step in bridging this gap is to redefine implementation (e.g., integration of findings and evidence into real-world practice) as the end-point of research, rather than stopping at dissemination (Morris, Wooding, \& Grant, 2011). Researchers should therefore routinely consider implementation at the design and proposal stage and investigate potential barriers to implementation, prior to investing significant time and resource. While this is requested by some funders already (e.g., the National Institute of Health Research in the UK), the need for researchers to adopt this practice as a matter of course is emphasised.

A second step towards successful translation is investing and engaging in strategies that maximise the success of real-world implementation in a sustainable and cost-effective manner (e.g., Kazdin \& Blase, 2011). The findings of the present study highlight the need for greater and earlier collaboration between researchers, practitioners, stakeholders, and users e.g., through scoping, acceptability studies, and public involvement. This can balance researcher desires with practical realities, to maximise successful implementation. As an exemplar, The Body Project is a cognitive-dissonance based eating disorder prevention program which is now implemented internationally in various formats (Becker et al., 2017), is supported by a train-the-trainer infrastructure, and shares practical resources for facilitators through an internationally accessible web platform. This success has arisen out of a dedication to effective implementation, and engagement with stakeholders in a community participatory approach (e.g., Becker, Stice, Shaw, \& Woda, 2009). Guidance may also be provided by the mixed methods action research framework (MMAR; Ivankova, 2014), recognising the need for both quantitative and qualitative work in generating solutions to real issues in practice.

In addition to engaging users and stakeholders in co-designing intervention pathways, the findings also call for closer collaboration between research and practice across clinical, health, and education settings more broadly. The authors urge a culture where practitioners and service users routinely receive information about research and its benefits, where research engagement is viewed as the norm rather than the exception, where data are collected as part of routine practice, and where research results and findings are made accessible to the public on a regular basis. Working in partnership as standard practice will foster the foundation for successful collaboration and therefore implementation.

Finally, there is a need to apply pressure to funders to include implementation as a phase of equal importance to others in the research process that needs resourcing. The increasing emphasis on impact (the contribution of research beyond academia), at least in the UK, has been a positive move in ensuring (or at least planning for) the translation of research (e.g., see the Research Excellence Framework [REF], http://www.ref.ac.uk/). However, there remains significant pressure from funding bodies for researchers to constantly innovate - proposing and evaluating novel theories and interventions in the search for bigger and better effects. Thus, 
existing theories and interventions are often left without further exploration (e.g., see Pennesi \& Wade, 2016), and therefore impact (including uptake and effectiveness) in practice remains largely unknown. We therefore need to create more pressure on traditional funders to allocate resource to implementation science, as well as consider non-traditional funding routes (e.g., alumni; relevant business, private and third-sector organisations; crowd-funding; aligning with corporate social responsibility agendas) to resource implementation efforts.

\subsection{Think big: positive and protective frameworks}

The final theme highlighted the need to expand the traditional focus on risk and negative factors with regard to body image and appearance, to better and more consistently include positive and protective factors. This enables a more holistic perspective to guide comprehensive theory, assessment, and intervention development and evaluation; and inform new opportunities to create broader social change. As a start, researchers and clinicians in all areas are exhorted to go beyond physical appearance, to consider broader conceptualisations of the body (e.g., functionality, attunement, embodiment, self-care) that encourage a more nuanced, integrative, and holistic approach to the body and appearance (see Tylka \& Piran, 2019). Given negative and positive body image are not simply two ends of a continuum (Tylka \& Wood-Barcalow, 2015), understanding and reducing negative body image - while serving the field well to date - can arguably lead to an absence of concerns and dissatisfaction at best, whereas an additional focus on positive body image can lead to a more holistic self-perception and enhanced well-being. Likewise, in the field of visible difference, broadening the focus from the appearance difference and assumed negative impacts, to include positive outcomes, adjustment, and resilience factors, reduces the pathologising of appearance and enables us to move towards a holistic conceptualisation and appreciation (Harcourt \& Williamson, 2019; Sharratt, Jenkinson, Moss, Clarke, \& Rumsey, 2018; Thomas et al., 2019). This is not intended to minimise or replace existing approaches (e.g., identifying and targeting risk factors) that play a crucial role in our understanding and interventions, but to complement them in maximising progress and impact.

The findings indicate that as a field we still have limited understanding of positive and protective factors at the level of theory development and assessment, despite the initiation of a number of positive intervention approaches (e.g., body functionality, yoga, mindfulness, and acceptance; Alleva, Holmqvist Gattario, Martijn, \& Lunde, 2019; Alleva, Martijn, Van Breukelen, Jansen, \& Karos, 2015; Atkinson \& Diedrichs, 2019; Cook-Cottone, Talebkhah, Guyker, \& Keddie, 2017; Cox \& McMahon, 2019; Griffiths, Williamson, Zucchelli, Paraskeva, \& Moss, 2018; Halliwell, Dawson, \& Burkey, 2019; Halliwell, Jarman, Tylka, \& Slater, 2018; Mulgrew, Prichard, Stalley, \& Lim, 2019), and the development and use of measures for some aspects of positive body image (see Webb et al., 2015). Further research is therefore required to improve developmental understanding of positive body image and resilience in various groups and settings, including how proposed positive and protective constructs and predictors interact with each other and with traditionally assessed negative outcomes (e.g., mediational and interactional explanatory models). In addition, closer collaboration between the fields of body image and visible difference (e.g., in developing and applying models, measures, and interventions across domains, e.g., Feragen \& Stock, 2018) could greatly benefit understanding and progress in both areas, as well as help to reduce the issue of multiple constructs, overlap, and reinventing the wheel noted above.

In addition to theory development, adopting a positive and protective framework for intervention development and evaluation is vital. This is perhaps particularly relevant for universal intervention approaches, which are provided to whole groups regardless of the existing level of risk and aimed at primary prevention of concerns. Traditionally, such interventions have been assessed on their capacity to reduce negative outcomes (e.g., body dissatisfaction and weight/shape concerns, internalisation of appearance ideals); however, there is an inherent problem in measuring risk reduction in a population that by definition is not yet evidencing risk. In so doing there is a danger of underestimating the effectiveness of an intervention, and prematurely discarding it. For example, universal prevention for eating disorders has traditionally shown smaller effect sizes than targeted interventions (Le, Barendregt, Hay, \& Mihalopoulos, 2017; Stice, Shaw, \& Marti, 2007), and as a result have often been neglected (Wilksch, 2014). The authors therefore highlight a necessary shift towards dual aims for interventions in our field to not only reduce negative outcomes, but encourage positive and protective outcomes (e.g., body appreciation, psychological flexibility, well-being; Piran, 2017; Stock, Feragen, Moss, \& Rumsey, 2018), and evaluate them accordingly. In addition to appropriate long-term follow-up spanning key developmental periods, a more holistic focus will inform appropriate measure selection to capture a broader range of benefits for existing programmes (Piran, 2015), as well as inform refinement and development of novel interventions. A more accurate picture of efficacy will additionally enable appropriate resource allocation and underpin policy decisions.

Finally, in conjunction with the first theme to go beyond the individual, it will also be important to research effective ways of facilitating constructive and positive processes with regard to communities and societies - where norms of respect for individuals inhabiting diverse bodies are widely accepted and can engender protection for future generations. One avenue is to assess and utilise existing social platforms and movements for communicating positive and protective themes. For example, in an experimental study comparing trends on social media - quickly gaining traction as a negative influence on body image - Slater, Varsani, and Diedrichs (2017) found that body positive social media (self-compassion quotes) attenuated the negative impacts of media on body satisfaction. Thus, identifying avenues for shifting or redirecting attention to existing positive aspects of popular culture, such as those offered by the body positive movement, could translate into measurable societal impact. As mentioned above, public engagement, activism and advocacy will also play an important and vital role in creating positive social change, and researchers and clinicians are urged to explore opportunities for engaging in this alongside research (see Table 1 for suggestions).

\subsection{Limitations}

Although the content of the presentations and ensuing thematic analysis emphasise priority areas for shaping future work in research translation, the authors recognise the limitations. First, the number of participants was low, and the presentations were short in duration; therefore, we had limited data from which to extract themes and examine prevalence. Second, the participants were selected from a pool of conference attendees by the last three authors. We do not claim that these presenters are representative of the entire field, and we recognise that the findings offer a particular perspective that may not be shared by all (e.g., applied research, social justice, advocacy, and activism). Nevertheless, co-chairs did aim to capture contributors from a range of backgrounds and levels of experience in the selection process, and the convergence of key themes would suggest the salience of our findings to a certain extent, and at the least, offer ideas for further consideration. However, participants were all working in high-income Western countries, predominantly reflected an applied research perspec- 
tive and endorsement of a social justice philosophy. The inclusion of researchers and clinicians working in other countries, and from more diverse backgrounds and disciplines could help expand our perspectives - not only when it comes to setting priorities for future research, but also with respect to, for example, identifying priorities for clinical practice, advocacy, and activism. As a related note, we highlight the role of the first two authors as both participants and those who conducted the analysis. While a rigorous analytic procedure was followed, including review by all speakers/authors, it is important to note this as the context for which to consider the above findings and discussion. Finally, the conference was held two years ago at the time of submission; however, the themes are still deemed to be relevant.

\subsection{Conclusion}

These findings offer timely considerations to move the field forward in engendering real progress in translating research to impact. Speakers called for discourse and interventions that target overarching systems rather than individuals, greater effort in consolidating constructs and methods currently utilised, closer collaboration at all stages of research and practice, an increased focus on implementation throughout the entire research process, and going beyond the traditional focus on negative body image to include a greater emphasis on encompassing positive and protective factors. Overall, the message is to 'think bigger.' While we may not yet have all the answers for all questions of interest, we do have enough research evidence to make a difference to society. We encourage researchers to make the leap and leave the academic comfort blankets behind more often, to broaden the focus of our field to engage with the 'big picture'; maximising our chances of success in stemming the tidal wave of body and appearance dissatisfaction and creating a world where all may flourish.

\section{Declaration of Competing Interest}

The Dove Self-Esteem Project (Dove, Unilever) was one of several sponsors of the Appearance Matters 7 Conference. Phillippa C. Diedrichs, Melissa Atkinson, Heidi Williamson, and Nichola Rumsey have received research and/or consultancy funding from Dove, Unilever. Dove had no input into the content of, or decision to publish, this manuscript.

\section{Acknowledgments}

The contribution from Jessica M. Alleva was supported by NWO Grant 446-15-011 "Handsome is as handsome does: Investigating body functionality and novel mechanisms of body image improvement," awarded to Jessica M. Alleva. Dr Nicola Stock was partially supported by The Scar Free Foundation at the time of Appearance Matters 7. The contribution of Dr. Niva Piran was supported by the Social Sciences and Humanities Research Council of Canada (grants \#410-2011-0205; 410-2007-0630; 410-2003-0280; 4101999-1370).

\section{References}

Alleva, J. M., Holmqvist Gattario, K., Martijn, C., \& Lunde, C. (2019). What can my body do vs. How does it look? A qualitative analysis of young women and men's descriptions of their body functionality or physical appearance. Body Image, 31, 71-80. http://dx.doi.org/10.1016/j.bodyim.2019.08.008

Alleva, J. M., Martijn, C., Van Breukelen, G. J., Jansen, A., \& Karos, K. (2015). Expand your Horizon: A programme that improves body image and reduces self-objectification by training women to focus on body functionality. Body Image, 15, 81-89. http://dx.doi.org/10.1016/j.bodyim.2015.07.001

Atkinson, M. J., \& Diedrichs, P. C. (2019). Brief cognitive interventions for improving positive body image and resilience to media exposure in young adult females. Manuscript in preparation.
Avalos, L. C., \& Tylka, T. L. (2006). Exploring a model of intuitive eating with college women. Journal of Counseling Psychology, 53, 486-497. http://dx.doi.org/10. 1037/0022-0167.53.4.486

Austin, S. B. (2015). Accelerating progress in eating disorders prevention: A call for policy translation research and training. Eating Disorders, 24, 6-19. http://dx. doi.org/10.1080/10640266.2015.1034056

Becker, C. B., Stice, E., Shaw, H., \& Woda, S. (2009). Use of empirically supported interventions for psychopathology: Can the participatory approach move us beyond the research-to-practice gap? Behaviour Research and Therapy, 47, 265-274. http://dx.doi.org/10.1016/j.brat.2009.02.007

Becker, C. B., Perez, M., Kilpela, L. S., Diedrichs, P. C., Trujillo, E., \& Stice, E. (2017). Engaging stakeholder communities as body image intervention partners: The Body Project as a case example. Eating Behaviors, 25, 62-67. http://dx.doi.org/ 10.1016/j.eatbeh.2016.03.015

Braun, V., \& Clarke, V. (2006). Using thematic analysis in psychology. Qualitative Research in Psychology, 3, 77-101, https://psycnet.apa.org/doi/10.1191/1478088706qp063oa.

Calogero, R. M. (2013). Objects don't object: Evidence that self-objectification disrupts women's social activism. Psychological Science, 24, 312-318, https://doi.org/10.1177\%2F0956797612452574.

Calogero, R. M., \& Jost, J. T. (2011). Self-subjugation among women: Sexist ideology, self-objectification, and the buffering function of the need to avoid closure. Journal of Personality and Social Psychology, 100, 211-228. http://dx. doi.org/10.1037/a0021864

Calogero, R. M., \& Tylka, T. L. (2014). Sanctioning resistance to sexual objectification: An integrative system justification perspective. The Journal of Social Issues, 70, 763-778. http://dx.doi.org/10.1111/josi.12090

Cash, T. F., \& Smolak, L. (2011). Body image: A handbook of science, practice, and prevention. New York, NY: Guilford Press.

Cohen, R., Irwin, L., Newton-John, T., \& Slater, A. (2019). \#bodypositivity: A content analysis of body positive accounts on Instagram. Body Image, 29, 47-57. http:/| dx.doi.org/10.1016/j.bodyim.2019.02.007

Cook-Cottone, C. (2006). The attuned representation model for the primary prevention of eating disorders: An overview for school psychologists. Psychology in the Schools, 43, 223-230. http://dx.doi.org/10.1002/pits.20139

Cook-Cottone, C., Talebkhah, K., Guyker, W., \& Keddie, E. (2017). A controlled trial of a yoga-based prevention program targeting eating disorder risk factors among middle school females. Eating Disorders, 25, 392-405. http://dx.doi.org/ $10.1080 / 10640266.2017 .1365562$

COST Action. (2017). Cost action final achievement report. IS1210: Appearance matters: Tackling the physical and psychosocial consequences of dissatisfaction with appearance. Brussels, Belgium: COST Association.

Cox, A. E., \& McMahon, A. K. (2019). Exploring changes in mindfulness and body appreciation during yoga participation. Body Image, 29, 118-121. http://dx.doi. org/10.1016/j.bodyim.2019.03.003

Craddock, N., Ramsey, M., Spotswood, F., Halliwell, E., \& Diedrichs, P. C. (2019). Can big business foster positive body image? Qualitative insights from industry leaders walking the talk. Body Image, 30, 93-106. http://dx.doi.org/10.1016/j. bodyim.2019.06.004

Diedrichs, P. C. (2016). Eating disorder prevention in the real-world. In T. Wade (Ed.), Encyclopedia of feeding and eating disorders. New York, NY: Springer. http://dx.doi.org/10.1007/978-981-287-087-2_133-1

Fardouly, J., Diedrichs, P. C., Vartanian, L. R., \& Halliwell, E. (2015). Social comparisons on social media: The impact of Facebook on young women's body image concerns and mood. Body Image, 13, 38-45. http://dx.doi.org/10.1016/j. bodyim.2014.12.002

Fardouly, J., \& Rapee, R. M. (2019). The impact of no-makeup selfies on young women's body image. Body Image, 28, 128-134. http://dx.doi.org/10.1016/j. bodyim.2019.01.006

Feragen, K. B., \& Stock, N. M. (2018). Factors affecting subjective appearance evaluations among patients with congenital craniofacial conditions: An application of Cash's cognitive-behavioural model of body image development Body Image, 24, 124-136. http://dx.doi.org/10.1016/j.bodyim.2017.12.005

Gill, R. (2007). Critical respect: The difficulties and dilemmas of agency and "choice" for feminism: A reply to Duits and van Zoonen. European Journal of Women's Studies, 14, 69-80. http://dx.doi.org/10.1177/1350506807072318

Griffiths, C., Williamson, H., Zucchelli, F., Paraskeva, N., \& Moss, T. (2018). A systematic review of the effectiveness of Acceptance and Commitment Therapy (ACT) for body image dissatisfaction and weight self-stigma in adults. Journal of Contemporary Psychotherapy, 48, 189-204. http://dx.doi.org/10.1007/ s10879-018-9384-0

Halliwell, E., Dawson, K., \& Burkey, S. (2019). A randomized experimental evaluation of a yoga-based body image intervention. Body Image, 28, 119-127. http://dx.doi.org/10.1016/j.bodyim.2018.12.005

Halliwell, E., Jarman, H., Tylka, T. L., \& Slater, A. (2018). Evaluating the impact of a brief yoga intervention on preadolescents' body image and mood. Body Image, 27, 196-201. http://dx.doi.org/10.1016/j.bodyim.2018.10.003

Harcourt, D., \& Williamson, H. (2019). Positive body image in people living with visible differences. In T. L. Tylka \& N. Piran (Eds.), Handbook of positive body image and embodiment: Constructs, protective factors, and interventions (pp. 255-262). New York, NY: Oxford University Press.

Hartman, Y., \& Darab, S. (2012). A call for slow scholarship: A case study on the intensification of academic life and its implications for pedagogy. The Review of Education Pedagogy \& Cultural Studies, 34(1-2), 49-60. http://dx.doi.org/10. 1080/10714413.2012.643740 
Holland, G., \& Tiggemann, M. (2016). A systematic review of the impact of the use of social networking sites on body image and disordered eating outcomes. Body Image, 17, 100-110. http://dx.doi.org/10.1016/j.bodyim.2016.02.008

Homan, K. J., \& Tylka, T. L. (2018). Development and exploration of the gratitude model of body appreciation in women. Body Image, 25, 14-22. http://dx.doi org/10.1016/j.bodyim.2018.01.008

Ivankova, N. V. (2014). Mixed methods applications in action research. New York, NY: Sage.

Jankowski, G. S. (2016). Who stops the sweatshops? Our neglect of the injustice of maldistribution. Social and Personality Psychology Compass, 10, 581-590. http: dx.doi.org/10.1111/spc3.12272

Kazdin, A. E., \& Blase, S. L. (2011). Rebooting psychotherapy research and practice to reduce the burden of mental illness. Perspectives on Psychological Science, 6 , 21-37. http://dx.doi.org/10.1177/1745691610393527

Le, L. K., Barendregt, J. J., Hay, P., \& Mihalopoulos, C. (2017). Prevention of eating disorders: A systematic review and meta-analysis. Clinical Psychology Review, 53, 46-58. http://dx.doi.org/10.1016/j.cpr.2017.02.001

Lonergan, A. R., Bussey, K., Mond, J., Brown, O., Griffiths, S., \& Murray, S. B., et al. (2019). Me, my selfie, and I: The relationship between editing and posting selfies and body dissatisfaction in men and women. Body Image, 28, 39-43. http://dx.doi.org/10.1016/j.bodyim.2018.12.001

Louis, W. R., Mavor, K. I., La Macchia, S. T., \& Amiot, C. E. (2014). Social justice and psychology: What is, and what should be. Journal of Theoretical and Philosophical Psychology, 34, 14-27. http://dx.doi.org/10.1037/a0033033

McVey, G. L., Levine, M. P., Piran, N. \& Ferguson, H. B. (Eds.). (2013). Preventing eating-related and weight-related disorders: Collaborative research, advocacy, and policy change. Ontario, Canada: Wilfrid Laurier University Press.

Massey, S. G., \& Barreras, R. E. (2013). Introducing “impact validity.”. The Journal of Social Issues, 69, 615-632. http://dx.doi.org/10.1111/josi.12032

Mills, J. S., Musto, S., Williams, L., \& Tiggemann, M. (2018). “Selfie” harm: Effects on mood and body image in young women. Body Image, 27, 86-92. http://dx.doi. org/10.1016/j.bodyim.2018.08.007

Morris, Z. S., Wooding, S., \& Grant, J. (2011). The answer is 17 years, what is the question: Understanding time lags in translational research. Journal of the Royal Society of Medicine, 104, 510-520. http://dx.doi.org/10.1258/jrsm.2011.110180

Mulgrew, K. E., Prichard, I., Stalley, N., \& Lim, M. S. C. (2019). Effectiveness of a multi-session positive self, appearance, and functionality program on women's body satisfaction and response to media. Body Image, 31, 102-111. http://dx. doi.org/10.1016/j.bodyim.2019.08.012

Nuffield Council on Bioethics. (2017). Cosmetic procedures: Ethical issues Available from. www.nuffieldbioethics.org/project/cosmetic-procedures

Paraskeva, N., Lewis-Smith, H., \& Diedrichs, P. C. (2017). Consumer opinion on social policy approaches to promoting positive body image: Airbrushed media images and disclaimer labels. Journal of Health Psychology, 22, 164-175, https://doi.org/10.1177\%2F1359105315597052.

Pennesi, J. L., \& Wade, T. D. (2016). A systematic review of the existing models of disordered eating: Do they inform the development of effective interventions? Clinical Psychology Review, 43, 175-192. http://dx.doi.org/10.1016/j.cpr.2015. 12.004

Pfitzer, M., Bockstette, V., \& Stamp, M. (2013). Innovating for shared value. Harvard Business Review, 91, 100-107

Piran, N. (2015). New possibilities in the prevention of eating disorders: The introduction of positive body image measures. Body Image, 14, 146-157. http:// dx.doi.org/10.1016/j.bodyim.2015.03.008

Piran, N. (2017). Journeys of embodiment at the intersection of body and culture: The Developmental Theory of Embodiment. San Diego, California: Elsevier Press.

Piran, N., Levine, M., \& Steiner-Adair, C. (1999). Preventing eating disorders: A handbook of intervention and special challenges. Philadelphia, PA: Brunner/Mazel.

Piran, N., \& Teall, T. (2012). The developmental theory of embodiment. In G. McVey, M. P. Levine, N. Piran \& H. B. Ferguson (Eds.), Preventing eating-related and weight-related disorders: Collaborative research, advocacy, and policy change (pp. 169-198). Ontario, Canada: Wilfrid Laurier University Press.

Porter, M., \& Kramer, M. (2006). Strategy and society: The link between corporate social responsibility and competitive advantage. Harvard Business Review, 84, 78-92.

Record, K. L., \& Austin, S. B. (2016). "Paris thin": A call to regulate life-threatening starvation of runway models in the US fashion industry. American Journal of Public Health, 106, 205-206, https://dx.doi.org/10.2105\%2FAJPH.2015.302950.
Riley, S., Burns, M., Frith, H. \& Markula, P. (Eds.). (2008). Critical bodies: Representations, practices and identities of weight and body management. London: Palgrave/MacMillan.

Riley, S., Evans, A., \& Mackiewicz, A. (2016). It's just between girls: Negotiating the postfeminist gaze in women's 'looking talk.'. Feminism \&' Psychology, 26 94-113, https://doi.org/10.1177\%2F0959353515626182.

Riley, S., Evans, A., \& Robson, M. (2018). Postfeminism and health. London: Routledge.

Rodgers, R. F., Kruger, L., Lowy, A. S., Long, S., \& Richard, C. (2019). Getting Real about body image: A qualitative investigation of the usefulness of the Aerie Real campaign. Body Image, 30, 127-134. http://dx.doi.org/10.1016/j.bodyim. 2019.06.002

Rumsey, N. \& Harcourt, D. (Eds.). (2012). Oxford handbook of the psychology of appearance. London: Oxford University Press.

Sharratt, N. D., Jenkinson, E., Moss, T., Clarke, A., \& Rumsey, N. (2018). Understandings and experiences of visible difference and romantic relationships: A qualitative exploration. Body Image, 27, 32-42. http://dx.doi. org/10.1016/j.bodyim.2018.08.002

Slater, A., Cole, N., \& Fardouly, J. (2019). The effect of exposure to parodies of thin-ideal images on young women's body image and mood. Body Image, 29, 82-89. http://dx.doi.org/10.1016/j.bodyim.2019.03.001

Slater, A., Varsani, N., \& Diedrichs, P. C. (2017). \#fitspo Or \#loveyourself? The impact of fitspiration and self-compassion Instagram images on women's body image, self-compassion, and mood. Body Image, 22, 87-96. http://dx.doi.org/ 10.1016/j.bodyim.2017.06.004

Stice, E., Shaw, H., \& Marti, C. N. (2007). A meta-analytic review of eating disorder prevention programs: Encouraging findings. Annual Review of Clinical Psychology, 3, 207-231. http://dx.doi.org/10.1146/annurev.clinpsy.3.022806. 091447

Stock, N. M., Feragen, K. B., Moss, T. P., \& Rumsey, N. (2018). Toward a conceptual and methodological shift in craniofacial research. The Cleft Palate-Craniofacial Journal, 55, 105-111, https://doi.org/10.1177\%2F1055665617721925.

Stock, N. M., Hammond, V., Owen, T., Kiff, J., Shanly, A., \& Rumsey, N. (2016). Achieving consensus in the measurement of psychological adjustment to cleft lip and/or palate. The Cleft Palate-Craniofacial Journal, 53, 421-426. http://dx. doi.org/10.1597/15-071

Thomas, E. V., Warren-Findlow, J., Webb, J. B., Quinlan, M. M., Laditka, S. B., \& Reeve, C. L. (2019). "It's very valuable to me that I appear capable": A qualitative study exploring relationships between body functionality and appearance among women with visible physical disabilities. Body Image, 30, 81-92. http://dx.doi.org/10.1016/j.bodyim.2019.05.007

Thompson, J. K., Heinberg, L. J., Altabe, M., \& Tantleff-Dunn, S. (1999). Exacting beauty: Theory, assessment, and treatment of body image disturbance. Washington, DC: American Psychological Association.

Tiggemann, M., Hayden, S., Brown, Z., \& Veldhuis, J. (2018). The effect of Instagram "likes" on women's social comparison and body dissatisfaction. Body Image, 26 90-97. http://dx.doi.org/10.1016/j.bodyim.2018.07.002

Tiggemann, M., \& Zinoviev, K. (2019). The effect of \#enhancement-free Instagram images and hashtags on women's body image. Body Image, 31, 131-138. http:/ dx.doi.org/10.1016/j.bodyim.2019.09.004

Tylka, T. L., \& Iannantuono, A. C. (2016). Perceiving beauty in all women: Psychometric evaluation of the Broad Conceptualization of Beauty Scale. Body Image, 17, 67-81. http://dx.doi.org/10.1016/j.bodyim.2016.02.005

Tylka, T. L. \& Piran, N. (Eds.). (2019). Handbook of positive body image and embodiment: Constructs, protective factors, and interventions. New York, NY: Oxford University Press.

Tylka, T. L., \& Wood-Barcalow, N. L. (2015). What is and what is not positive body image? Conceptual foundations and construct definition. Body Image, 14, 118-129. http://dx.doi.org/10.1016/j.bodyim.2015.04.001

Open Science Collaboration. (2015). Estimating the reproducibility of psychological science. Science, 349(6251) http://dx.doi.org/10.1126/science.aac4716

Webb, J. B., Wood-Barcalow, N. L., \& Tylka, T. L. (2015). Assessing positive body image: Contemporary approaches and future directions. Body Image, 14, 130-145. http://dx.doi.org/10.1016/j.bodyim.2015.03.010

Wilksch, S. M. (2014). Where did universal eating disorder prevention go? Eating Disorders, 22, 184-192. http://dx.doi.org/10.1080/10640266.2013.864889

Yager, Z., Diedrichs, P. C., Ricciardelli, L. A., \& Halliwell, E. (2013). What works in secondary schools? A systematic review of classroom-based body image programs. Body Image, 10, 271-281. http://dx.doi.org/10.1016/j.bodyim.2013. 04.001 